\title{
FACTORIAL PROPERTY OF A RING OF AUTOMORPHIC FORMS
}

\author{
SHIGEAKI TSUYUMINE
}

\begin{abstract}
A ring of automorphic forms is shown to be factorial under some conditions on the domain and on the Picard group. As an application, we show that any divisor on the moduli space $\mathfrak{M}_{g}$ of curves of genus $g \geqslant 3$ is defined by a single element, and that the Satake compactification of $\mathfrak{M}_{g}$ is written as a projective spectrum of a factorial graded ring. We find a single element which defines the closure of $\mathfrak{M}_{4}^{\prime}$ in $\mathfrak{M}_{4}$ where $\mathfrak{M}_{4}^{\prime}$ is the moduli of curves of genus four whose canonical curves are exhibited as complete intersections of quadric cones and of cubics in $\mathbb{P}^{3}$.
\end{abstract}

Introduction. Let $X$ be a normal quasi-projective variety over $\mathbb{C}$ such that (i) $X$ has a normal projective compactification $X^{*}$ with $\operatorname{codim}\left(X^{*}-X\right) \geqslant 2$, and (ii) $X$ is a quotient space $\mathscr{D} / \Gamma$ of a connected domain $\mathscr{D}$ with $\operatorname{Pic}(\mathscr{D})=0$, by a discontinuous group $\Gamma$ of holomorphic automorphisms. Then the (canonically determined) homogeneous coordinate ring of $X$ is factorial under some conditions on $\Gamma$, or essentially on the Picard group $\mathrm{Pic}^{c} \Gamma$ in our notation (Theorem 1). When $\mathscr{D}$ is the Siegel space and $\Gamma$ is the modular group, Freitag $[9,10]$ has shown that the homogeneous coordinate ring of $X$, or more precisely the graded ring of Siegel modular forms, is factorial, provided that the degree is greater than two.

Our theorem is applicable to the case when $X$ is the moduli space $\mathfrak{M}_{g}$ of curves of genus $g$. We need to know the first or second Betti number of $\mathfrak{M}_{g}$ or of the Teichmüller modular group $\Gamma_{g}$, which will "almost" determine the Picard group. In Mumford [19], the first Betti number $b_{1}\left(\mathfrak{M}_{g}\right)$ of $\mathfrak{M}_{g}$ was shown to be 0 , and it was conjectured that the second Betti number $b_{2}\left(\mathfrak{M}_{g}\right), g \geqslant 3$, would be one. Harer [12] proved it for $g \geqslant 5$, and in this paper we show it for $g=3$, 4 , which seems, more or less, well known. As for the case of $\mathscr{D}$ being a bounded symmetric domain, the determination of the Betti numbers is covered by the calculation of the stable real cohomology due to Borel [2] for a fairly large class of $\mathscr{D}$. As an application of our method we show that the closure in $\mathfrak{M}_{4}$, of the moduli $\mathfrak{M}_{4}^{\prime}$ of nonhyperelliptic curves of genus four exhibited as complete intersections of quadric cones and of cubics in $\mathbb{P}^{3}$ is a subvariety defined by a principal ideal, and gives the generator of the principal ideal.

This work was done while the author was staying at Harvard University. He wishes to express his hearty thanks to the members of the Department of Mathematics of Harvard University for their hospitality, and to the Educational Project for

Received by the editors March 7, 1985 and, in revised form, August 19, 1985.

1980 Mathematics Subject Classification. Primary 10D20; Secondary 14H10.

Key words and phrases. Automorphic form, Picard group, Betti number, moduli of curves, theta constant, Schottky invariant.

(1986 American Mathematical Society $0002-9947 / 86 \$ 1.00+\$ .25$ per page 
Japanese Mathematical Scientists for financial support. He wishes to thank A. Yukie for useful conversations and for informing him which cohomology groups are calculated, and which are not, and also to the referee for his comments, especially that on the Satake compactification of $\mathfrak{M}_{g}$.

\section{Automorphic forms.}

1.1. Let $\mathscr{D}$ be a connected open domain in $\mathbb{C}^{m}$, and let $\Gamma$ be a group of holomorphic automorphisms acting properly discontinuously on $\mathscr{D}$. The quotient $X=\mathscr{D} / \Gamma$ is a normal analytic space. We shall denote by $\pi$ the canonical projection of $\mathscr{D}$ to $X$.

The map $\rho: \Gamma \times \mathscr{D} \rightarrow \mathbb{C}^{\times}$is called a (holomorphic) automorphy factor if $\rho(\gamma, z)$ is holomorphic in $z \in \mathscr{D}$ for any fixed $\gamma \in \Gamma$ and satisfies

$$
\rho\left(\gamma \gamma^{\prime}, z\right)=\rho\left(\gamma, \gamma^{\prime} z\right) \rho\left(\gamma^{\prime}, z\right) \text { for } \gamma, \gamma^{\prime} \in \Gamma \text {. }
$$

$\rho$ is said to be trivial if there is $u \in \Gamma\left(\mathscr{D}, \mathcal{O}_{\mathscr{D}}^{\times}\right)$such that $\rho(\gamma, z)=u(\gamma z) / u(z)$, where $\mathcal{O}_{\mathscr{D}}^{\times}$denotes the subsheaf of the structure sheaf $\mathcal{O}_{\mathscr{D}}$ consisting of the invertible elements. We shall denote by Pic $\Gamma$ the group of equivalence classes of automorphy factors mod trivial ones. Pic $\Gamma$ is isomorphic to the cohomology group $H^{1}\left(\Gamma, \mathcal{O}_{\mathscr{D}}^{\times}\right)$by the definition. Let $L$ denote the subgroup of Pic $\Gamma$ which is composed of $\rho$ such that $\rho(\gamma, z)$ equals one for any pair $(\gamma, z)$ of $\gamma \in \Gamma$ and of its fixed point $z$. If $\Gamma$ acts freely on $\mathscr{D}$, then $L$ equals $\operatorname{Pic} \Gamma$. Further let $\operatorname{Pic}(X)$ denote the group of analytic invertible sheaves mod linear equivalence, and $\operatorname{Pic}^{c}(X)$ its subgroup whose elements are corresponding to Cartier divisors, where a divisor means a locally finite formal sum $\sum n_{i} D_{i}$, with $n_{i} \in \mathbb{Z}$, of irreducible analytic subsets $D_{i}$ of codimension one, and a Cartier divisor means a divisor locally defined by a single meromorphic function.

For an automorphy factor $\rho$ there is the coherent sheaf $\mathscr{U}_{\rho}$ on $X$ defined by

$$
\Gamma\left(U, \mathscr{U}_{\rho}\right)=\left\{f \in \Gamma\left(\pi^{-1}(U), \mathcal{O}_{\mathscr{D}}\right) \mid f(\gamma z)=\rho(\gamma, z) f(z), \gamma \in \Gamma, z \in \pi^{-1}(U)\right\},
$$

$U$ being any open subset of $X$. The global section of $\mathscr{U}_{\rho}$ is called the $\rho$-automorphic form. We note that $\mathscr{U}_{\rho}$ is actually invertible if $\rho$ represents an element of $L$.

1.2. The different choice of $\rho$ in one equivalence class makes $\mathscr{U}_{\rho}$ stable mod linear equivalence. So we have a homomorphism of $L$ to $\operatorname{Pic}(X)$. However we have the inverse also, under the condition $\operatorname{Pic}(\mathscr{D})=0$.

Lemma 1. Suppose $\operatorname{Pic}(\mathscr{D})=0$. Then $L$ and $\operatorname{Pic}(X)$ are canonically isomorphic, so $\operatorname{Pic}(X)$ is a subgroup of Pic $\Gamma$.

Proof. Let $\mathscr{U} \in \operatorname{Pic}(X)$. Then $\pi^{*} \mathscr{U}$ is an invertible sheaf on $\mathscr{D}$. By our assumption there is an isomorphism of $\mathcal{O}_{\mathscr{D}}$ to $\pi * \mathscr{U}$. Let $f$ be the image of $1 \in \mathcal{O}_{\mathscr{D}}$. Then $\rho(\gamma, z)=f(\gamma z) / f(z)$ gives an automorphy factor. The different choice of the isomorphisms makes $\rho$ stable mod trivial factors. $\rho$ is obviously in $L$. Thus we have given the inverse of $\rho \mapsto \mathscr{U}_{\rho}$. Q.E.D.

Let Lemma 1 be the case. Let us define $\operatorname{Pic}^{c} \Gamma$ by

$$
\operatorname{Pic}^{c} \Gamma=\left\{\rho \in \operatorname{Pic} \Gamma \mid \rho^{m} \in \operatorname{Pic}^{c}(X) \text { for some nonzero } m\right\} \text {. }
$$


We have the following diagam of inclusions:

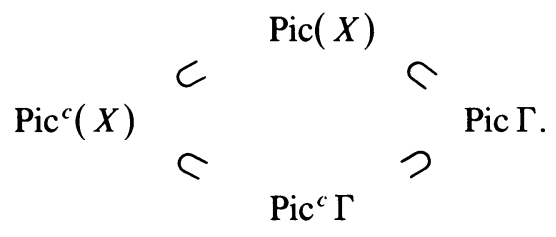

The next lemma is easy to show. However it is a key lemma in our method.

Lemma 2. Suppose Pic(D) $=0$. Take any effective divisor $D$ on $X$. Then there is $\rho \in \operatorname{Pic} \Gamma$ and a $\rho$-automorphic form $f$ such that the divisor $(f)$ on $\mathscr{D}$ is equal to the divisor $\pi^{-1}(D)$.

Proof. $\pi^{-1}(D)$ is locally finite. Since $\mathscr{D}$ is nonsingular, such a divisor is expressed as a Cartier divisor. Since $\operatorname{Pic}(\mathscr{D})=0$, the second problem of Cousin is solvable for $\mathscr{D}$, and hence there is a holomorphic function $f$ such that $(f)=\pi^{-1}(D)$. Since $\pi^{-1}(D)$ is $\Gamma$-invariant, $f(\gamma z) / f(z)$ is holomorphic and vanishes nowhere. So $\rho(\gamma, z)$ $=f(\gamma z) / f(z)$ is an automorphy factor. $f$ is obviously a $\rho$-automorphic form. Q.E.D.

\section{Factorial property.}

2.0. Till the end of the next section, we keep the following assumption and notations.

AsSUMPTION. Let $X$ be a normal quasi-projective variety, and let $X^{*}$ be a normal projective variety having $X$ as its Zariski open subset with $\operatorname{dim} X^{*} \geqslant \operatorname{dim}\left(X^{*}-X\right)$ +2 . We assume that $X$ as a complex space is written as the quotient $\mathscr{D} / \Gamma$ of a connected open domain $\mathscr{D}$ with $\operatorname{Pic}(\mathscr{D})=0$, by a group $\Gamma$ of holomorphic automorphisms acting properly discontinuously. Further, if the order of a fixed point $z \in \mathscr{D}$ is defined to be the order of the stabilizer subgroup of $\Gamma$ at $z$, then we assume that the orders of all the fixed points are bounded (e.g., $\Gamma$ has a subgroup of finite index which acts freely on $\mathscr{D}$ ).

Notations. We denote by $\pi$ the canonical projection of $\mathscr{D}$ to $X=\mathscr{D} / \Gamma$. $\mathcal{O}_{X}$ denotes the analytic structure sheaf. $X^{*}$ carries an ample invertible sheaf $\mathscr{L}_{0}$, which we shall fix. Since $X^{*}$ is normal and projective, it equals $\operatorname{Proj}\left(\oplus_{k \geqslant 0} \Gamma\left(X^{*}, \mathscr{L}_{0}^{k}\right)\right)$. There is an automorphy factor corresponding to $\left.\mathscr{L}_{0}\right|_{X}$, say $\mu_{0} \in L \subset \operatorname{Pic} \Gamma$. We take an automorphy factor $\mu$ such that $\mu^{l_{0}}=\mu_{0}$ for some $l_{0} \geqslant 1$. Let us fix such $\mu$ as makes $l_{0}$ maximum if it exists. A $\mu^{k}$-automorphic form is said to be an automorphic form of weight $k$, and let $\mathscr{U}(k)$ denote the coherent sheaf $\mathscr{U}_{\mu^{k}}$ on $X$ of automorphic forms of weight $k . \mathscr{L}(k)$ denotes the extension $i_{*} \mathscr{U}(k), i$ being the inclusion map of $X$ into $X^{*}$.

$\mathscr{L}\left(l_{0}\right)$ equals $\mathscr{L}_{0}$ (Serre [23]). Obviously we have

$$
\begin{aligned}
& \mathscr{L}(k) \otimes \mathscr{L}\left(k^{\prime}\right) \subset \mathscr{L}\left(k+k^{\prime}\right) \quad \text { for any } k, k^{\prime} \geqslant 0, \\
& \mathscr{L}(k) \otimes \mathscr{L}\left(l_{0}\right)=\mathscr{L}\left(k+l_{0}\right) \quad \text { for any } k \geqslant 0 .
\end{aligned}
$$


$\Gamma\left(X^{*}, \mathscr{L}(k)\right)$ is finite dimensional since obviously so is $\Gamma\left(X^{*}, \mathscr{L}_{0}^{k}\right)$ and since $\Gamma\left(X^{*}, \mathscr{L}(k)\right)^{\otimes I_{0}} \subset \Gamma\left(X^{*}, \mathscr{L}_{0}^{k}\right)$. Moreover we have

$$
X^{*}=\operatorname{Proj}\left(\bigoplus_{k \geqslant 0} \Gamma\left(X^{*}, \mathscr{L}(k)\right)\right) .
$$

$\mathscr{L}_{0}$ corresponds to some divisor, hence so is $\left.\mathscr{L}_{0}\right|_{x}$. Hence $\mu$ is actually in $\operatorname{Pic}^{c} \Gamma$. In particular, Pic ${ }^{c} \Gamma$ has a subgroup isomorphic to $\mathbb{Z}$. There is no difference between algebraic divisors on $X$ and analytic ones by Remmert's theorem and by Chow's theorem. So $\operatorname{Pic}^{c}(X)$ is nothing other than the Picard group in the algebraic sense.

2.1. Let $\mathscr{D}$ be a bounded symmetric domain. $\mathscr{D}$ is written as a quotient space $G(\mathbb{R})^{0} / K$ where $G(\mathbb{R})^{0}$ is the identity component of real points of a semisimple algebraic group $G$ over $\mathbb{Q}$, and $K$ is its maximal compact subgroup. Let us take an arithmetic subgroup $\Gamma$. Then there is a natural compactification $X^{*}$ of $X=\mathscr{D} / \Gamma$ which is called the Satake, or Baily-Borel compactification. Suppose that $G$ has no normal $\mathbb{Q}$-subgroup of dimension three with $\mathbb{Q}$-rank one. Then $\operatorname{dim} X^{*} \geqslant$ $\operatorname{dim}\left(X^{*}-X\right)+2$. In this case, our assumption is fully satisfied. For the details we refer to Baily and Borel [1].

Let $j(\gamma, z)$ be the Jacobian of $\gamma \in \Gamma$ at $z \in \mathscr{D}$. Then $j$ is in Pic $\Gamma$. There is an integer $r$ such that the sheaf $\mathscr{U}_{j^{-r}}$ on $X$ extends to an ample invertible sheaf $\mathscr{L}_{0}$ on $X^{*}$ (loc. cit.). For the sake of simplicity, let us suppose that Pic $\Gamma$ is finitely generated. Then $\mu$ (determined in 2.0 ) is given as an automorphy factor such that

$$
\mu^{l}=j^{-1}
$$

where $l$ is the maximum in every choice of such $\mu$. However, $\mu$ is not necessarily unique, indeed there will be ambiguity caused by characters of $\Gamma$ which are killed by lth power.

2.2. Our purpose here is to show Theorem 1 . We need two lemmas.

Lemma 3. There is a nonzero integer $N$ such that $N \cdot \operatorname{Pic} \Gamma \subset \operatorname{Pic}(X), N \cdot \operatorname{Pic}^{c} \Gamma \subset$ $\operatorname{Pic}^{c}(X)$. In particular, $\operatorname{Pic}(X)\left(\right.$ resp. $\left.\operatorname{Pic}^{c}(X)\right)$ is of finite index in $\operatorname{Pic} \Gamma\left(\right.$ resp. $\left.\operatorname{Pic}^{c} \Gamma\right)$ if they are finitely generated.

Proof. Let $z$ be a fixed point of order $m$. Then by definition, any automorphy factor takes an $m$ th root of unity at $(\gamma, z)$ with any $\gamma$ fixing $z$. So if $N$ is the least common multiple of the orders of all the fixed points, then $\rho^{N}$ must be in $L$ for any $\rho \in \operatorname{Pic} \Gamma, L$ being as in 1.1. By Lemma 1 our assertion follows. Q.E.D.

LeMma 4. Let $\rho$ be the automorphy factor determined in Lemma 2. Then $\rho \in \operatorname{Pic}^{c} \Gamma$.

Proof. Let $D, \rho, f$ be as in Lemma 2. Let $N$ be as in Lemma 3, and let $E=N D$, $\sigma=\rho^{N}, g=f^{N}$. It is easy to see that $E$ is a Cartier divisor of $X$ by using the fact that (i) $\pi^{-1}(E)$ is defined by $g$ on $\mathscr{D}$ and that (ii) $\sigma \in L$. The sheaf $\mathscr{U}_{\sigma}$ on $X$, corresponding to $\sigma$ is invertible and has a global section $g$ whose divisor is equal to $E$. Hence $\mathscr{U}_{\sigma}$ is corresponding to the divisor $E$. So $\sigma \in \operatorname{Pic}^{c}(X)$, and hence $\rho \in \operatorname{Pic}^{\prime} \Gamma$. Q.E.D. 
THEOREM 1. Let the assumption be as in (2.0). Suppose $\operatorname{Pic}^{c} \Gamma \cong \mathbb{Z}$. Then any effective divisor on $X^{*}$ is defined by a single element, i.e., it is a zero of some automorphic form. Moreover the graded ring $\oplus_{k \geqslant 0} \Gamma\left(X^{*}, \mathscr{L}(k)\right)$ of automorphic forms is factorial.

Proof. Let $D^{*}$ be any effective divisor of $X^{*}$. Then $D^{*}$ is equal to the closure of $D=D^{*} \cap X$ in $X^{*}$ in the strong topology. Lemma 2 shows that there exists a $\rho$-automorphic form $f$ for some $\rho$ such that the divisor $(f)$ on $\mathscr{D}$ is equal to $\pi^{-1}(D)$. Since $\rho \in \operatorname{Pic}^{c} \Gamma$ by Lemma 4 and since $\operatorname{Pic}^{c} \Gamma \cong \mathbb{Z}, \rho$ is equivalent to some $\mu^{k} \bmod$ trivial automorphy factors. So we may assume that $f$ is an automorphic form of weight $k$ (by replacing $f$ by the product of $f$ and of some $u \in \Gamma\left(\mathscr{D}, \mathcal{O}_{\mathscr{D}}^{\times}\right)$). Then the divisor $(f)$ on $X$ equals $D$, and hence $(f)$ on $X^{*}$ coincides with $D^{*}$. This proves the first assertion.

Now let us take an automorphic form $f . f$ is a prime element if and only if the divisor $(f)$ on $X$ is prime. Then if $f \mid g h$ for some automorphic forms $f, g$, then we get $f \mid g$ or $f \mid h$, considering the corresponding divisors. So any homogeneous element of the graded ring $\oplus_{k \geqslant 0} \Gamma\left(X^{*}, \mathscr{L}(k)\right)$ is uniquely factored into a product of prime homogeneous elements up to scalar factors. Then the ring is factorial by the theorem of Samuel [21]. Q.E.D.

REMARK. Even if $X^{*}$ is given by $X^{*}=\operatorname{Proj}(A)$ with a factorial graded ring, $X^{*}$ may not be a locally factorial variety. However if $A$ is generated by elements of degree one, then so is $X^{*}$.

\section{Cohomological calculation.}

3.1. We shall look for some sufficient condition for $\operatorname{Pic}^{c} \Gamma$ to be isomorphic to $\mathbb{Z}$, or equivalently that $\operatorname{Pic}^{c} \Gamma$ is torsion free and that $\operatorname{dim}_{\mathbb{R}} \operatorname{Pic}^{c} \Gamma \otimes_{Z} \mathbb{R}=1$. Our argument is partly similar to that of Matsushima [18, §10], where the case of $X$ being nonsingular and compact is treated. The last condition is equivalent to $\operatorname{dim}_{\mathbf{R}} \operatorname{Pic}^{c}(X) \otimes_{\mathbf{Z}} \mathbb{R}=1$ by Lemma 3. Since $\operatorname{dim}_{\mathbf{R}} \operatorname{Pic}^{c} \Gamma \otimes_{\mathbf{Z}} \mathbb{R}>0$, it is also a sufficient condition for this that $\operatorname{dim}_{\mathbf{R}} \operatorname{Pic}(X) \otimes_{\mathbf{Z}} \mathbb{R}$ or $\operatorname{dim}_{\mathbf{R}} \operatorname{Pic} \Gamma \otimes_{\mathbf{Z}} \mathbb{R}$ equals one.

Let us consider the short exact sequences of two kinds;

$$
\begin{aligned}
& 0 \rightarrow \mathbb{Z} \rightarrow \mathcal{O}_{\mathscr{D}} \stackrel{\exp }{\rightarrow} \mathcal{O}_{\mathscr{D}}^{\times} \rightarrow 0, \\
& 0 \rightarrow \mathbb{Z} \rightarrow \mathcal{O}_{X} \stackrel{\exp }{\rightarrow} \mathcal{O}_{X}^{\times} \rightarrow 0 .
\end{aligned}
$$

From these we derive the long exact sequences

$$
\begin{aligned}
& \cdots \rightarrow H^{1}(\Gamma, \mathbf{Z}) \rightarrow H^{1}\left(\Gamma, \mathcal{O}_{\mathscr{D}}\right) \rightarrow \operatorname{Pic} \Gamma \rightarrow H^{2}(\Gamma, \mathbb{Z}) \rightarrow \cdots, \\
& \cdots \rightarrow H^{1}(X, \mathbf{Z}) \rightarrow H^{1}\left(X, \mathcal{O}_{X}\right) \rightarrow \operatorname{Pic}(X) \rightarrow H^{2}(X, \mathbb{Z}) \rightarrow \cdots .
\end{aligned}
$$

We shall denote by $b_{i}(\Gamma), b_{i}(X)$, the Betti number of $\Gamma, X$ (i.e., $\operatorname{dim}_{\mathbf{R}} H^{i}(\Gamma, \mathbb{R})$, $\left.\operatorname{dim}_{\mathbb{R}} H^{i}(X, \mathbb{R})\right)$ respectively. When $H^{i}\left(\Gamma, \mathcal{O}_{\mathscr{D}}\right)$ (resp. $\left.H^{i}\left(X, \mathcal{O}_{X}\right)\right)$ vanishes, $\operatorname{dim}_{\mathbf{R}} \operatorname{Pic} \Gamma \otimes_{\mathbf{Z}} \mathbb{R}$ (resp. $\operatorname{dim}_{\mathbf{R}} \operatorname{Pic}(X) \otimes_{\mathbf{Z}} \mathbb{R}$ ) is bounded from above by the second Betti number. 
As for the torsion part of $\mathrm{Pic}^{c} \Gamma$ we have the following

Lemma 5. Suppose that the abelian quotient group $\Gamma /[\Gamma, \Gamma]$ is finite. Then the torsion part of Pic $\Gamma$ is isomorphic to $\Gamma /[\Gamma, \Gamma]$. Especially if $\Gamma /[\Gamma, \Gamma]$ is trivial, then $\operatorname{Pic} \Gamma$ is torsion free, hence so are $\operatorname{Pic}^{c} \Gamma, \operatorname{Pic}(X), \operatorname{Pic}^{c}(X)$.

Proof. Any character of $\Gamma$ is an automorphy factor which is torsion in $\operatorname{Pic}^{c} \Gamma$. Conversely let $\rho \in \operatorname{Pic} \Gamma$ be a torsion element of order $m$. We may assume $\rho^{m}$ is identically equal to one. So $\rho$ is a constant in $z$. The map of $\Gamma$ to $\mathbb{C}^{\times}$given by $\gamma \mapsto \rho(\gamma, z)$ is a character. So there is an isomorphism between the torsion part of Pic $\Gamma$ and the character group of $\Gamma$ which is dual to $\Gamma /[\Gamma, \Gamma]$. Q.E.D.

3.2. By Lemma 3 we have $\operatorname{dim}_{\mathbb{C}} H^{1}\left(\Gamma, \mathcal{O}_{\mathscr{D}}\right)=\operatorname{dim}_{\mathbb{C}} H^{1}\left(X, \mathcal{O}_{X}\right)$ (both of them can be infinity). On the other hand, if $\mathscr{D}$ is acyclic (i.e., all the higher homology groups vanish) and if $\Gamma$ acts freely on $\mathscr{D}$, then the cohomology groups $H^{i}(\Gamma, \mathbb{Z})$ and $H^{i}(X, \mathbb{Z})$ are isomorphic for any $i$ (cf. Mac Lane [17, Chapter IV, 11]), hence $b_{i}(\Gamma)=b_{i}(X)$. However the coincidence of the Betti numbers follows from a weaker condition, for example this is the case if $\Gamma$ has a normal subgroup of finite index acting freely on $\mathscr{D}$ (for example, see the argument in Borel and Wallach [3, VIII, 2.2]). We note that contractible spaces (e.g., bounded symmetric domains, the Teichmüller space) are acyclic.

Proposition 1. Suppose $\operatorname{dim} X^{*} \geqslant \operatorname{dim}\left(X^{*}-X\right)+3$. Then both $H^{1}\left(\Gamma, \mathcal{O}_{\mathscr{D}}\right)$ and $H^{1}\left(X, \mathcal{O}_{X}\right)$ vanish in the following cases:

(1) There is an analytic subset $S$ of codimension $\geqslant 3$ containing all the singularities such that $b_{1}(X-S)=0$.

(2) $\Gamma$ has a normal subgroup $\Gamma^{\prime}$ of finite index acting freely on $\mathscr{D}$ such that $b_{1}\left(\mathscr{D} / \Gamma^{\prime}\right)=0$.

(3) $\mathscr{D}$ is simply connected, and $\Gamma$ has a normal subgroup $\Gamma^{\prime}$ of finite index acting freely on $\mathscr{D}$ such that the abelian quotient group $\Gamma^{\prime} /\left[\Gamma^{\prime}, \Gamma^{\prime}\right]$ is finite.

\subsection{Proof of Proposition 1. At first we show the following}

Lemma. Let $V$ be a nonsingular variety described as a projective variety minus subvarieties of codimension $\geqslant 3$. Then if $b_{1}(V)=0$, then $H^{1}\left(V, \mathcal{O}_{V}\right)$ vanishes.

Proof. We may assume that $V$ is an open subvariety of a normal projective variety. Our assumption implies that any invertible sheaf on $V$ extends to the projective variety as a coherent sheaf (Siu [24]). In particular, it is an algebraic invertible sheaf on $V$ (Serre [23]), and so $\operatorname{Pic}(V)=\operatorname{Pic}^{c}(V)$. By the general theory we can take a smooth projective variety $\bar{V}$ containing $V$ as a Zariski open subset such that $\bar{V}-V$ is a divisor with only normal crossing (cf. Deligne [6, 3.2]). We have a surjective homomorphism of $\operatorname{Pic}^{c}(\bar{V})$ to $\operatorname{Pic}^{c}(V)$ by the restriction of divisors, hence of $\operatorname{Pic}(\bar{V})$ to $\operatorname{Pic}(V)$. So, to prove our assertion, it is enough to show the vanishing of $H^{1}\left(\bar{V}, \mathcal{O}_{\bar{V}}\right)$. By Deligne [6, Corollary (3.2.14)], $b_{1}(V)=0$ implies that there are no holomorphic 1-forms on $\bar{V}$ (more strongly no meromorphic 1-forms on $V$ with logarithmic poles along $\bar{V}-V)$. So, by Hodge theory $H^{1}\left(\bar{V}, \mathcal{O}_{\bar{V}}\right)$ vanishes. Q.E.D. 
Proof of (1). Let $H_{S}^{i}\left(X, \mathcal{O}_{X}\right)$ denote the local cohomology group with support $S$. Then we have an exact sequence

$$
\cdots \rightarrow H_{S}^{1}\left(X, \mathcal{O}_{X}\right) \rightarrow H^{1}\left(X, \mathcal{O}_{X}\right) \rightarrow H^{1}\left(X-S, \mathcal{O}_{X-S}\right) \rightarrow \cdots .
$$

Since $X$ is normal and $S$ is of codimension $\geqslant 2, H_{S}^{1}\left(X, \mathcal{O}_{X}\right)$ vanishes by Serre [23]. By the above lemma $H^{1}\left(X-S, \mathcal{O}_{X-S}\right)$ also vanishes. This proves (1).

Proof of (2). Let $Y=\mathscr{D} / \Gamma^{\prime}$ and let $Y^{*}$ be the normalization of $X^{*}$ in the rational function field of $Y$. Then $Y, Y^{*}$ satisfy our assumption (2.0) and

$$
\operatorname{dim} Y^{*} \geqslant \operatorname{dim}\left(Y^{*}-Y\right)+3 .
$$

By (1), $H^{1}\left(Y, \mathcal{O}_{Y}\right)$ vanishes. If $\phi$ denotes the canonical projection of $Y$ to $X$, then $H^{1}\left(X, \mathcal{O}_{X}\right)$ is a subspace of $H^{1}\left(X, \phi_{*} \mathcal{O}_{Y}\right)$ which is isomorphic to $H^{1}\left(Y, \mathcal{O}_{Y}\right)$ by the Leray spectral sequence. So $H^{1}\left(X, \mathcal{O}_{X}\right)$ vanishes.

Proof of (3). Since $\mathscr{D}$ is simply connected, the fundamental group $\pi_{1}(Y)$ is just $\Gamma^{\prime}$. By the Hurewicz theorem $H_{1}(Y, \mathbb{Z})$ is isomorphic to $\Gamma^{\prime} /\left[\Gamma^{\prime}, \Gamma^{\prime}\right]$, hence $H_{1}(Y, \mathbb{Z})$ is of free rank 0 . The duality theorem for homology and cohomology groups shows that $H^{1}(Y, \mathbb{R})$ vanishes, i.e., $b_{1}(Y)=0$. So this case is reduced to (2). Q.E.D.

3.5. Summing up, we have the following

THEOREM 1'. The assertion of Theorem 1 holds in the following cases:

(i)(a) $\Gamma=[\Gamma, \Gamma]$, and (b) at least one of $\operatorname{Pic}^{c}(X), \operatorname{Pic}(X), \operatorname{Pic}^{c} \Gamma, \operatorname{Pic} \Gamma$ is a $\mathbb{Z}$-module of rank one.

(ii)(a) $\Gamma=[\Gamma, \Gamma]$, and (b) one of (1), (2), (3) in Proposition 1 together with $\operatorname{dim} X^{*} \geqslant \operatorname{dim}\left(X^{*}-X\right)+3$, and $(\mathrm{c}) b_{2}(\Gamma)$ or $b_{2}(X)=1$.

We note that for a large number of classes of arithmetic subgroups $\Gamma$ of semisimple Lie groups, the conditions that $b_{1}(\Gamma)=0$ and that $b_{2}(\Gamma)=1$ are satisfied (Borel [2]). For example, the Siegel modular group $\operatorname{Sp}_{2 g}(\mathbb{Z}), g \geqslant 4$, satisfies them. Further we have $\operatorname{Sp}_{2 g}(\mathbb{Z})=\left[\operatorname{Sp}_{2 g}(\mathbb{Z}), \operatorname{Sp}_{2 g}(\mathbb{Z})\right]$ for $g \geqslant 3$ (cf. Maass [16]), and so the condition (ii) in Theorem $1^{\prime}$ is satisfied. This shows that any divisor on the Siegel modular variety of degree $g \geqslant 4$ is defined by a principal ideal and that the ring of Siegel modular forms of degree $\geqslant 4$ is factorial, since $\mu$ (defined in 2.1) is given by

$$
\mu(M, Z)= \begin{cases}|C Z+D|^{2} & \text { if } g \text { is odd, }>1, \quad M=\left(\begin{array}{ll}
A & B \\
C & D
\end{array}\right) \in \operatorname{Sp}_{2 g}(\mathbb{Z}), \\
|C Z+D| & \text { if } g \text { is even, }\end{cases}
$$

$Z$ being a point of the Siegel space (Christian [5]). This has been proven by Freitag $[9,10]$. The same is true also for $g=3$ (loc. cit.), which we show in the next section in terms of the moduli space of curves.

\section{Moduli space of curves.}

4.1. Let $\mathfrak{M}_{g}$ denote the moduli space of curves of genus $g$ defined over $\mathbb{C}$. Let $\mathscr{A}_{g}$ denote the moduli space of principally polarized abelian varieties of dimension $g$ and defined over $\mathbb{C}$, and $\mathscr{A}_{g}^{*}$ its Satake compactification. The Torelli map $\mathfrak{M}_{g} \rightarrow \mathscr{A}_{g}$ gives an immersion. Let $\mathfrak{M}_{g}^{*}$ denote the normalization of the closure of $\mathfrak{M}_{g}$ in $\mathscr{A}_{g}^{*}$, which we call the Satake compactification of $\mathfrak{M}_{g} \cdot \mathfrak{M}_{g}^{*}$ has $\mathfrak{M}_{g}$ as its Zariski open 
subset and if $g \geqslant 3$, then $\mathfrak{M}_{g}^{*}-\mathfrak{M}_{g}$ is of codimension $2 . \mathfrak{M}_{g}$ is given as the quotient space $T_{g} / \Gamma_{g}$ of the Teichmüller space $T_{g}$ by the Teichmüller modular group $\Gamma_{g}$. If $g \geqslant 2$, then $T_{g}$ is a bounded domain in $\mathbb{C}^{3 g-3}$ which is contractible. $\Gamma_{g}$ acts properly discontinuously on $T_{g}$, and the orders of fixed points are bounded (because the orders of the automorphism groups of curves of a fixed genus $g>1$ are bounded). The abelian quotient group $\Gamma_{g} /\left[\Gamma_{g}, \Gamma_{g}\right]$ is known to be trivial for $g \geqslant 3$ (Powell [20]). Moreover Harer proved that $\operatorname{Pic}^{c}\left(\mathfrak{M}_{g}\right)$ is isomorphic to $\mathbb{Z}$ for $g \geqslant 5$ ([12], see also [13, p. 86]). We shall show this also for $g=3,4$ below. It seems to be more or less well known, but the references are hard to find. This is equivalent to $b_{2}\left(\mathfrak{M}_{g}\right)=1$ by Mumford [19].

We state the above as a proposition.

Proposition 2. $\operatorname{Pic}^{c}\left(\mathfrak{M}_{g}\right) \cong \mathbb{Z}$ for $g \geqslant 3$.

4.2. As a corollary to Theorem 1 we have the following

TheOREM 2. Let $g \geqslant 3$. Then $\mathfrak{M}_{g}^{*}$ is written as $\mathfrak{M}_{g}^{*}=\operatorname{Proj}(A)$ where $A=\bigoplus_{k \geqslant 0} A_{k}$ is a factorial graded ring. Moreover for any effective divisor $D$ on $\mathfrak{M}_{g}^{*}$ there is some $f \in A_{k}$ for some $k>0$ such that the divisor $(f)$ equals $D$.

4.3. We shall prepare to prove Proposition 2 for $g=3,4$. It is enough to show $\operatorname{dim}_{\mathbf{R}} \operatorname{Pic}\left(\mathfrak{M}_{g}\right) \otimes_{\mathbf{Z}} \mathbb{R} \leqslant 1$. For a variety $V$, let $\mathrm{Cl}(V)$ denote the algebraic Weil divisor class group. If $V$ is normal and if it is exhibited as a projective variety minus subvarieties of codimension $\geqslant 2$, then we have $\operatorname{Pic}^{c}(V) \subset \mathrm{Cl}(V)$. So Proposition 2 follows if we show $\operatorname{dim}_{\mathbf{R}} \mathrm{Cl}\left(\mathfrak{M}_{g}\right) \otimes_{\mathbf{Z}} \mathbb{R} \leqslant 1$ for $g=3,4$. Here we collect some results related to a divisor class group.

LeMMA 6. (i) Let $V$ be a normal variety, and $V^{\prime}$ be its open subvariety. If $V-V^{\prime}$ is of codimension $\geqslant 2$, then $\mathrm{Cl}(V) \cong \mathrm{Cl}\left(V^{\prime}\right)$.

(ii) Let $V, V^{\prime}$ be as above. Suppose that $\sum D_{i}=V-V^{\prime}$ is a divisor. Then we have an exact sequence

$$
\oplus \mathbb{Z} \cdot D_{i} \rightarrow \mathrm{Cl}(V) \rightarrow \mathrm{Cl}\left(V^{\prime}\right) \rightarrow 0 .
$$

(iii) Let $V$ be a normal projective variety with $\mathrm{Cl}(V)=\mathbb{Z}+\{$ finite group $\}$, and let $D$ be any effective divisor. Then $\mathrm{Cl}(V-D)$ is finite.

(iv) Let $V^{\prime}$ be a normal subvariety of an affine variety, and $V$ be a projective variety having $V^{\prime}$ as its open subset. Then $V-V^{\prime}$ is of codimension one.

(v) Let $\tilde{V} \rightarrow V$ be a finite morphism of normal varieties. Then $\operatorname{dim}_{\mathbf{R}} \mathrm{Cl}(V) \otimes_{\mathbf{Z}} \mathbb{R} \leqslant$ $\operatorname{dim}_{\mathbf{R}} \mathrm{Cl}(\tilde{V}) \otimes_{\mathbf{Z}} \mathbb{R}$.

(vi) Let $R$ be a noetherian graded $\mathbb{C}$-domain, and $G$ be a connected semisimple affine group acting $\mathbb{C}$-linearly on $R$ so as to preserve degrees. If $R$ is factorial, then so is the invariant subring $R^{G}$.

The proof is easy, and so we skip it (as for (vi), see Forgaty [8, Exercise V-6]).

4.4. Proof for $g=3$. Let $\mathfrak{M}_{3}^{\prime}$ be the moduli space of the hyperelliptic curves of genus three, and $\mathfrak{M}_{3}^{0}$ be that of the nonhyperelliptic curves of genus three. We have 
$\mathfrak{M}_{3}^{0}=\mathfrak{M}_{3}-\mathfrak{M}_{3}^{\prime}$, which is affine. Any curve in $\mathfrak{M}_{3}^{0}$ can be exhibited as a nonsingular quartic in $\mathbb{P}^{2}$, and hence it has a ternary quartic as its defining equation. Hence $\mathfrak{M}_{3}^{0}$ is an affine open subset of $\operatorname{Proj}(S(3,4)$ ) where $S(3,4)$ is the graded ring of invariants of ternary quartics, i.e., the invariant subring of the polynomial ring in 15 variables under the action of $\mathrm{Sl}_{3}(\mathbb{C})$ in the well-known manner. By Lemma 6(vi), (iv), (iii), (i), $\mathrm{Cl}\left(\mathfrak{M}_{3}^{0}\right)$ is finite, and thus $\operatorname{dim}_{\mathbf{R}} \mathrm{Cl}\left(\mathfrak{M}_{3}\right) \otimes_{\mathbf{Z}} \mathbb{R} \leqslant 1$ by Lemma 6(ii) since $\mathfrak{M}_{3}^{\prime}$ is an irreducible divisor of $\mathfrak{M}_{3}$. Q.E.D.

4.5. Proof for $g=4$. Let $C$ be a nonhyperelliptic nonsingular curve of genus four. We identify $C$ with its canonical curve in $\mathbb{P}^{3}$, which is a complete intersection of a quadric and of a cubic. There are two possibilities for a quadric in $\mathbb{P}^{3}$, that is, a smooth quadric or a quadric cone. It is known that the latter is the case if and only if some theta constant with even theta characteristic vanishes at a point of the Siegel space corresponding to the Jacobian of $C$. Let $\mathfrak{M}_{4}^{0}$ be the moduli space of curves in the former case. $\mathfrak{M}_{4}^{0}$ is an affine open subset of $\mathfrak{M}_{4}$. A smooth quadric is unique up to automorphisms of the projective space $\mathbb{P}^{3}$, and hence curves in $\mathfrak{M}_{4}^{0}$ will be classified by means of the corresponding cubics.

Here we adapt the argument by Catanese [4], to which we refer for the detail and for the references. Employing $y={ }^{t}\left(y_{0}, \ldots, y_{3}\right)$ as homogeneous coordinates of $\mathbb{P}^{3}$, let $\Lambda$ be the space of $3 \times 3$ symmetric matrices whose entries are linear forms $l_{i j}(y)$ in $y_{0}, \ldots, y_{3}$. The determinant of a nonsingular matrix of $\Lambda$ defines a cubic $G$, which is called a symmetric cubic. Such $G$ is uniquely determined projectively by the equivalence class of $\Lambda \bmod \mathrm{GL}_{3}(\mathbb{C})$ under the action of $M \rightarrow{ }^{t} g M g, M \in \Lambda$, $g \in \mathrm{GL}_{3}(\mathbb{C})$, however the converse is also true (loc. cit., Theorem 1.3). Let $Q_{0}$ be a smooth quadric whose equation, we may (and do) assume, is normalized to the form $y_{0}^{2}+\cdots+y_{3}^{2}$. Let $\mathrm{PO}_{4}(\mathbb{C})$ be the subgroup of the automorphism group $\mathrm{PGL}_{4}(\mathbb{C})$ of $\mathbb{P}^{3}$, stabilizing $Q_{0}$ projectively. Let $R_{4}$ be the Prym moduli space of genus four, i.e., the moduli of double covers of curves of genus four, in other words, the moduli of pairs $(C, \eta), C \in \mathfrak{M}_{4}, 0 \neq \eta \in \operatorname{Pic}(C)_{2}$ (two torsion points). $R_{4}$ is a covering of $\mathfrak{M}_{4}$ of degree 255 . Let $\tilde{\mathfrak{M}}_{4}^{0}$ be the inverse image of the affine variety $\mathfrak{M}_{4}^{0} \cdot(C, \eta)$ is called bielliptic if $C$ is a double cover $\pi: C \rightarrow E$ of an elliptic curve and $\eta=\pi^{*} \eta^{\prime}$ for $\eta^{\prime} \in \operatorname{Pic}(E)_{2}$. The locus of bielliptic curves is of codimension three. Let $U$ denote the open subset of the affine variety $\tilde{\mathfrak{M}}_{4}^{0}$ which is the complement of the bielliptic locus. Then $U$ is described as the quotient by $\mathrm{PO}_{4}(\mathbb{C})$, of $\{$ symmetric cubics $G \subset \mathbb{P}^{3} \mid G$ is normal and $C=G \cap Q_{0}$ is a smooth curve) (loc. cit., Theorem 1.5, see also the proof of Theorem 1.18).

Regarding $G$ as an element of the equivalence class $\Lambda \bmod \mathrm{GL}_{3}(\mathbb{C}), U$ is an open subvariety of $\mathbb{P}^{23} / \mathrm{PGL}_{3}(\mathbb{C}) \times \mathrm{PO}_{4}(\mathbb{C})$ where $\mathbb{P}^{23}=\Lambda-\{0\} / \mathbb{C}^{\times}$and $\mathrm{PGL}_{3}(\mathbb{C})$, $\mathrm{PO}_{4}(\mathbb{C})$ act on $\mathbb{P}^{23}$ via $M \rightarrow t g M g, M \in \Lambda, g \in \mathrm{GL}_{3}(\mathbb{C})$, and $M=\left(l_{i j}(y)\right) \rightarrow$ $\left(l_{i j}(h y)\right), h \in \mathrm{GL}_{4}(\mathbb{C})$ representing $\bar{h} \in \mathrm{PO}_{4}(\mathbb{C})$, these two actions being commuting. The above actions induce those of $\mathrm{SL}_{3}(\mathbb{C}), \mathrm{SO}_{4}(\mathbb{C})$ via the canonical isogeny $\mathrm{SL}_{3}(\mathbb{C}) \rightarrow \mathrm{PGL}_{3}(\mathrm{C}), \mathrm{SO}_{4}(\mathbb{C}) \rightarrow \mathrm{PO}_{4}(\mathbb{C})$. Applying Lemma 6(vi) to our case, we get $\mathrm{CL}\left(\mathbb{P}^{23} / \mathrm{PGL}_{3}(\mathbf{C}) \times \mathrm{PO}_{4}(\mathbb{C})\right) \cong \mathbf{Z}$. By Lemma 6(iv), (iii), (i), $\mathrm{Cl}(U)$ is finite, and then by Lemma $6(\mathrm{i}),(\mathrm{v}), \mathrm{Cl}\left(\mathfrak{M}_{4}^{0}\right)$ is also finite. Hence $\mathrm{Cl}\left(\mathfrak{M}_{4}\right)$ is a $\mathbb{Z}$-module of rank one. Q.E.D. 


\section{Moduli spaces related to curves of genus four.}

5.1. At first we introduce theta functions. Let $k^{\prime}, k^{\prime \prime}$ be column vectors of size $g$ with rational numbers as their entries, and let $x={ }^{t}\left(x_{1}, \ldots, x_{g}\right) \in \mathbb{C}^{g}$. We define a theta function of degree $g$ with characteristic $k=\left(\begin{array}{c}k^{\prime} \\ k^{\prime \prime}\end{array}\right)$ by

$$
\theta[k](Z, x)=\sum_{r \in \mathbb{Z}^{g}} \mathbf{e}\left(\frac{1}{2}^{t}\left(r+k^{\prime}\right) Z\left(r+k^{\prime}\right)+{ }^{t}\left(r+k^{\prime}\right)\left(x+k^{\prime \prime}\right)\right)
$$

where $Z$ is a point of the Siegel space $H_{g}=\left\{Z \in M_{g}(\mathbb{C}) \mid{ }^{t} Z=Z, \operatorname{Im} Z>0\right\}$, and $\mathbf{e}(*)$ stands for $\exp (2 \pi \sqrt{-1} *)$. If $k \in\left(\frac{1}{2} \mathbb{Z} / \mathbb{Z}\right)^{2 g}$ and $\mathbf{e}\left(\frac{1}{2}^{t} k^{\prime} k^{\prime \prime}\right)=1$ (resp. -1$)$, then $k$ is said to be an even (resp. odd) theta characteristic. There are $2^{g-1}\left(2^{g}+1\right)$ even theta characteristics, and $2^{g-1}\left(2^{g}-1\right)$ odd ones. If $k$ is even, we put $\theta[k](Z)=$ $\theta[k](Z, 0)$, and call it a theta constant of degree $g$.

Proposition 3. Let $g \geqslant 3$, and let $N=2^{g-2}\left(2^{g}+1\right)$. The product

$$
\chi_{N}=\prod_{k: \text { even }} \theta[k]
$$

of all the theta constants with even theta characteristics is a Siegel modular form of weight $N$, and is a prime element of the ring of Siegel modular forms.

Proof. As for the first assertion, we refer to Igusa [14]. When $g=3, \chi_{18}$ is known to be a modular form whose divisor gives the hyperelliptic locus (loc. cit.), and hence it is a prime element. Let $g \geqslant 4$. Let

$$
\Gamma_{g}(1,2)=\left\{M=\left(\begin{array}{ll}
A & B \\
C & D
\end{array}\right) \mid \text { diagonal elements of }{ }^{t} A C,{ }^{t} B D \text { are } \equiv 0 \bmod 2\right\} .
$$

Then $\theta[0](Z)$ is a modular form for $\Gamma_{g}(1,2)$ of weight $\frac{1}{2}$ with a multiplier, and its divisor is irreducible in $H_{g} / \Gamma_{g}(1,2)$ (Endres [7] for $g \geqslant 8$, Freitag [11, 3.3. Proposition], for the general case). Let $f$ be any Siegel modular form for $\operatorname{Sp}_{2 n}(\mathbb{Z})$ which is a factor of $\chi_{N}$. Then the above implies that one of $f, \chi_{N} / f$ is divisible by $\theta[0]$ as holomorphic functions on $H_{g}$. We may assume that $f$ is divisible by $\theta[0]$. Then $f$ is obviously divisible by $\theta[k]$ for any even $k$. Since $\theta[k]$ 's have distinct divisors on $H_{g}$ to each other, $\chi_{N} \mid f$, and $f$ equals $\chi_{N}$ up to scalar factors. Thus $\chi_{N}$ is a prime element. Q.E.D.

5.2. Let $C$ be a curve in $\mathfrak{M}_{3}^{0}$, i.e., a smooth nonhyperelliptic curve of genus three. Let us identify $C$ with its canonical curve in $\mathbb{P}^{2}$. Then $C$ is a smooth quartic, and has exactly 28 bitangents, which correspond to the odd theta characteristics of degree three. Let $\tau_{1}, \tau_{2}, \tau_{3}$ be homogeneous coordinates of $\mathbb{P}^{2}$, and let $P(\tau)$ denote the quartic defining $C$. For all the odd theta characteristics $k_{i}(1 \leqslant i \leqslant 28)$ of degree three, $l_{i}$ 's denotes the line in $\mathbb{P}^{2}$ defined by

$$
l_{i}: l_{i}(\tau)=\sum_{j=1}^{3} \frac{\partial}{\partial x_{j}} \theta\left[k_{i}\right]\left(Z_{3}, 0\right) \tau_{j}, \quad 1 \leqslant i \leqslant 28,
$$

where $Z_{3} \in H_{3}$. If $Z_{3}$ corresponds to the Jacobian of $C$, then $l_{i}$ 's form all the bitangents to $C$. If $s$ denotes the image of $\prod_{i=1}^{28} l_{i}$ in the homogeneous coordinate ring $\mathbb{C}\left[\tau_{1}, \ldots, \tau_{3}\right] / P(\tau)$ of $C$, then $s$ vanishes only at $C \cap l_{1}, \ldots, C \cap l_{28}$, where the orders of vanishing are all two. In particular, there is no element $s^{\prime}$ in the homogeneous coordinate ring such that $s^{\prime \nu}=s, \nu>2$. 
Let $M$ be a subgroup of $\left(\frac{1}{2} \mathbb{Z} / \mathbb{Z}\right)^{8}$ of order 8 , and let $m_{1}, m_{2}, m_{3}$ be even theta characteristics with odd $m_{1}+m_{2}+m_{3}$ such that all the elements of $M+m_{1}$, $M_{2}+m_{2}, M_{3}+m_{3}$ are even. Put $r_{i}=\Pi_{m \in M+m_{i}} \theta[m]$. If $Z \in H_{4}$ is a Jacobian point, then we have

$$
\sqrt{r_{1}(Z)}+\sqrt{r_{2}(Z)}+\sqrt{r_{3}(Z)}=0 \quad \text { (Schottky's theta relation), }
$$

or equivalently, putting $J=r_{1}^{2}+r_{2}^{2}+r_{3}^{2}-2 r_{1} r_{2}-2 r_{2} r_{3}-2 r_{3} r_{1}$,

$$
J(Z)=0 \text {. }
$$

$J$ is determined up to $M, m_{1}, m_{2}, m_{3}$, and it is a cusp form of weight eight. For the proof of the above facts we refer to Schottky [22], Igusa [15]. $J$ is called the Schottky invariant. Let us decompose $Z \in H_{4}$ into

$$
Z=\left(\begin{array}{cc}
Z_{3} & \tau \\
{ }^{t} \tau & z
\end{array}\right), \quad Z_{3} \in H_{3}, z \in H_{1}, \tau={ }^{t}\left(\tau_{1}, \tau_{2}, \tau_{3}\right) \in \mathbb{C}^{3} .
$$

For $m=\left(\begin{array}{c}m^{\prime} \\ m^{\prime \prime}\end{array}\right) \in\left(\frac{1}{2} \mathbb{Z} / \mathbb{Z}\right)^{8}$, let $k=\left(\begin{array}{c}k^{\prime} \\ k^{\prime \prime}\end{array}\right) \in\left(\frac{1}{2} \mathbb{Z} / \mathbb{Z}\right)^{6}, k_{1}=\left(\begin{array}{c}k_{1}^{\prime} \\ k_{1}^{\prime \prime}\end{array}\right)$ be such that

$$
m^{\prime}=\left(\begin{array}{l}
k^{\prime} \\
k_{1}^{\prime}
\end{array}\right), \quad m^{\prime \prime}=\left(\begin{array}{l}
k^{\prime \prime} \\
k_{1}^{\prime \prime}
\end{array}\right)
$$

If $m$ is even, then $k, k_{1}$ are even or odd alike. Then

$$
\begin{aligned}
\theta[m](Z) & =\theta[k]\left(Z_{3}\right) \theta\left[k_{1}\right](z) \\
+ & \frac{1}{2 \pi \sqrt{-1}}\left(\sum_{j=1}^{3} \frac{\partial}{\partial x_{j}} \theta[k]\left(Z_{3}, 0\right) \tau_{j}\right) \frac{\partial}{\partial x} \theta\left[k_{1}\right](z, 0)+(\text { higher terms of } \tau) .
\end{aligned}
$$

Lemma 7. Let us expand the Schottky invariant $J$ in terms of $\tau$. Then the leading term is of the form

$$
P(\tau)\left(\theta\left[\begin{array}{l}
0 \\
0
\end{array}\right](z) \theta\left[\begin{array}{l}
0 \\
1
\end{array}\right](z) \theta\left[\begin{array}{l}
1 \\
0
\end{array}\right](z)\right)^{4}
$$

where $P(\tau)$ is a homogeneous polynomial in $\tau$ of degree four whose coefficients are polynomials in theta constants of degree three and in derivatives of theta functions of degree three at $x=0 . P(\tau)$ defines the canonical curve of genus three corresponding to $Z_{3} \in H_{3}$ if $Z_{3}$ is a Jacobian point of a nonhyperelliptic curve.

Proof. Let $k$ be an odd theta characteristic of degree three, and let $m=$ ${ }^{t}\left({ }^{t} k^{\prime}, \frac{1}{2},{ }^{t} k^{\prime \prime}, \frac{1}{2}\right)$, which is even. We may assume that $\theta[m]$ is a member of $r_{1}$, and moreover that also $r_{2}, r_{3}$ have, as their factors, theta constants with such characteristics. Then the first assertion is easy to verify. By definition of $J$ it follows that $\sum_{j=1}^{3} \frac{\partial}{\partial x_{j}} \theta[k]\left(Z_{3}, 0\right) \tau_{j}$ gives the bitangent to the curve defined by $P(\tau)$. Since $k$ is arbitrary, the curve defined by $P(\tau)$ has 28 bitangents which are $l_{1}, \ldots, l_{28}$. So if $Z_{3}$ is in the general position, $P(\tau)$ defines a smooth curve of genus three which corresponds to $Z_{3}$ by using the fact that $l_{1}, \ldots, l_{28}$ determine uniquely the quartic having them as bitangents (cf. Weber [25]). Q.E.D.

5.3. $\mathfrak{M}_{4}$ is defined by the Schottky invariant $J$ in $\mathscr{A}_{4}$ (Schottky [22], Igusa [15], Freitag [10]). It is known that at least one theta constant $\theta[m]$, even $m \in\left(\frac{1}{2} \mathbb{Z} / \mathbb{Z}\right)^{8}$, vanishes at a point of $\mathfrak{M}_{4}^{\prime}=\mathfrak{M}_{4}-\mathfrak{M}_{4}^{0} \subset \mathscr{A}_{4}=H_{4} / \operatorname{Sp}_{8}(\mathbb{Z})$. So the closure of $\mathfrak{M}_{4}^{\prime}$ 
in $\mathscr{A}_{4}$ is set-theoretically defined by the ideal $\left(J, \chi_{68}\right)$ where $\chi_{68}$ is as in 5.1 . $\left(J, \chi_{68}\right)$ is not a prime ideal. Indeed for a general point $P$ of $\mathfrak{M}_{4}^{\prime}$, just one theta constant vanishes at $P$. We may assume that it is a factor of $r_{1}$. Then in the local ring of $\mathfrak{M}_{4}$ at $P, r_{2}-r_{3}$ is in the ideal defining $\mathfrak{M}_{4}^{\prime}$, but not in the ideal generated by $J, \chi_{68}$.

THEOREM 3. Let $\mathfrak{M}_{4}^{\prime *}$ be the closure in $\mathfrak{M}_{4}^{*}$, of the subset whose points represent the curves exhibited as complete intersections of quadric cones and of cubics in $\mathbb{P}^{3}$. Then $\mathfrak{M}_{4}^{*}$ is defined by $\sqrt{\left.\chi_{68}\right|_{\mathfrak{M}_{4}^{*}}}$ in $\mathfrak{M}_{4}^{*}$.

$\sqrt{\chi_{68}}$ is not well defined as a holomorphic function on $H_{4}$ as Proposition 3 shows, but $\sqrt{\chi_{68} \mid \mathfrak{M}_{4}^{*}}$ is (see the next proof). It is not known if $\mathfrak{M}_{4}^{\prime}$ is a complete intersection in $\mathscr{A}_{4}$, or equivalently there is a modular form of weight 34 whose restriction to $\mathfrak{M}_{4}$ equals $\sqrt{\chi_{68} \mid \mathfrak{M}_{4}}$.

Proof of Theorem 3. Since $\mathfrak{M}_{4}^{\prime *}$ is a divisor on $\mathfrak{M}_{4}^{*}$, by Theorem 2 there is the automorphic form $f$ on $\mathfrak{M}_{4}$ whose divisor equals $\mathfrak{M}_{4}^{\prime *}$. Since $\left.\chi_{68}\right|_{\mathfrak{M}_{4}^{*}}$ vanishes only at $\mathfrak{M}_{4}^{\prime *}$, we may assume that $f^{\nu}=\left.\chi_{68}\right|_{\mathfrak{M}_{4}^{*}} \nu$ is greater than one, indeed the ideal $\left(J, \chi_{68}\right)$ would be prime if $\nu=1$. Expanding $\chi_{68}$ in terms of $\tau$, we have

$$
\chi_{68}(Z)=Q\left(Z_{3}, z\right) \prod_{i=1}^{28} l_{i}(\tau)+(\text { higher terms of } \tau)
$$

where $l_{i}(\tau)$ is as in 5.2 and $Q\left(Z_{3}, z\right)$ is a nontrivial function of $Z_{3}, z$. Let us fix $Z_{3}$, $z$ so that $Q\left(Z_{3}, z\right) \neq 0$ and $Z_{3}$ corresponds to a nonhyperelliptic curve of genus three. Let $P(\tau)$ be also as in 5.2. Then $f$ can be expanded in terms of $\tau$, where $\tau$ is under the relation $P(\tau)=0$. Let $s^{\prime}$ be its leading term, in other words, the homogeneous polynomial in $\tau$ of the lowest degree appearing in the expansion of $f$ which is not $\equiv 0 \bmod P(\tau)$. As elements of the homogeneous coordinate ring $\mathbb{C}\left[\tau_{1}, \tau_{2}, \tau_{3}\right] / P(\tau)$, we have $s^{\prime \nu}=s$ by Lemma 7 where $s$ denotes the image of $\Pi_{i=1}^{28} l_{i}(\tau)$. However $\nu \leqslant 2$ as we saw in 5.2, hence $\nu=2$, and the proof is complete. Q.E.D.

\section{REFERENCES}

1. W. L. Baily and A. Borel, Compactification of arithmetic quotients of bounded symmetric domains, Ann. of Math. (2) 84 (1966), 442-528.

2. A. Borel, Stable real cohomology of arithmetic groups. II, Manifolds and Lie Groups, Birkhäuser, 1981, pp. 21-55.

3. A. Borel and N. Wallach, Continuous cohomology, discrete subgroups and representations of reductive groups, Ann. of Math. Stud., no. 94, Princeton Univ. Press, Princeton, N.J., 1980.

4. F. Catanese, On the rationality of certain moduli spaces related to curves of genus 4, Lecture Notes in Math., vol. 1008 , Springer-Verlag, 1984 , pp. 30-50.

5. U. Christian, Über Hilbert-Siegelsche Modulformen und Poincarésche Reihen, Math. Ann. 148 (1962), 257-307.

6. P. Deligne, Théorie de Hodge. II, Inst. Hautes Études Sci. Publ. Math. 40 (1972), 5-52.

7. R. Endres, Multiplikatorsysteme der symplektische Thetagruppe, Monatsh. Math. 94 (1982), 281-297.

8. J. Forgaty, Invariant theorey, Math. Lecture Note Series, Benjamin, New York, 1969.

9. E. Freitag, Stable Modulformen, Math. Ann. 230 (1977), 197-211.

10. Die Irreducibilität der Schottky relation (Bemerkungen zu einem Satz von J. Igusa), Arch. Math. 40 (1983), 255-259.

11 , Holomorphic tensors on subvarieties of the Siegel modular variety, Automorphic forms of several variables (Taniguchi sympos., Katata, 1983), Birkhäuser, 1984, pp. 93-113. 
12. J. Harer, The second homology group of the mapping class group of an orientable surface, Invent. Math. 72 (1983), 221-239.

13. J. Harris and D. Mumford, On the Kodaira dimension of the moduli space of curves, Invent. Math. 67 (1982), 23-86.

14. J. Igusa, Modular forms and projective invariants, Amer. J. Math. 89 (1967), 817-855.

15. On the irreducibility of Schottky's divisor, J. Fac. Sci. Univ. Tokyo Sect. IA Math. 28 (1981), 531-545.

16. H. Maass, Die Multiplikatorsysteme zur Siegelschen Modulgruppe, Nachr. Akad. Wiss. Göttingen 11 (1964), 125-135.

17. S. Mac Lane, Homology, Grundlehren Math. Wiss., Band 114, Springer-Verlag, Berlin and New York, 1967.

18. Y. Matsushima, On the Betti numbers of compact, locally symmetric Riemannian manifolds, Osaka Math. J. 14 (1962), 1-20.

19. D. Mumford, Abelian quotients of the Teichmüller modular group, J. Analyse Math. 18 (1967), 227-244.

20. J. Powell, Two theorems on the mapping class group on a surface, Proc. Amer. Math. Soc. 68 (1978), 347-350.

21. P. Samuel, Sur les anneaux factoriels, Bull. Soc. Math. France 89 (1961), 155-173.

22. F. Schottky, Zur Theorie der Abelschen Funktionen von vier Variablen, J. Reine Angew. Math. 102 (1888), 304-352.

23. J.-P. Serre, Prolongement de faiceaux analytique coherents, Ann. Inst. Fourier (Grenoble) 16 (1966), $363-374$.

24. Y.-T. Siu, Extending coherent analytic sheaves, Ann. of Math. (2) 90 (1969), 108-143.

25. H. Weber, Theorie der Abel'schen Funktionen vom Geschlecht 3, Berlin, 1876.

2856 - 235, Sashiogi, OhmiYa - Shi, Saitama, 330, JAPan

Current address: Sonderforschungsbereich 170, Mathematisches Institut, Bunsenstrasse 3-5, 3400 Göttingen, West Germany 